\title{
NANOSCALE ENGINEERING WITH A TEM FOR DNA SEQUENCING
}

\author{
David C. Bell ${ }^{1,2}$ Min Jun Kim³ ${ }^{3}$ Meni Wanunu ${ }^{3}$, Amit Meller ${ }^{3}$ \\ ${ }^{1}$ Center for Nanoscale Systems \\ ${ }^{2}$ Division of Engineering and Applied Sciences, Harvard University, Cambridge, MA 02138 \\ ${ }^{3}$ Rowland Institute at Harvard, Cambridge, MA 02142
}

A $200 \mathrm{KV}$ Field Emission Transmission Electron Microscope has been successfully employed to fabricate various sizes of solid-state nanopores in a $50 \mathrm{~nm}$ thick silicon nitride membrane (Fig. 1). This process incorporates feedback to the fabrication process to acquire dimensional controls over synthesized pores at the single nanometer length scale.

By using this fabrication process we have developed two novel DNA sequencing approaches; measurement of an ionic current blockage and a parallel optical readout of DNA electrophoretically driven through single and hundreds solid-state nanoscale size pores (Fig. 4). This parallelism is achieved by the fabrication of a high density solid-state array of nanopores. Optical readout in arrays circumvents the direct electrical addressing of each pore. We will not only discuss our progress in both approaches and their application in ultra fast DNA sequencing but also present a new nanofabrication technique to form nanoscale pores in $50 \mathrm{~nm}$ thick $\mathrm{Si}_{3} \mathrm{~N}_{4}$ membrane using a controlled Scanning Transmission Electron Microscope (STEM) based nano-machining technique.

The dynamics of the observed drilling and shrinking of nanopores is determined by the break-down of the covalent bonds and the surface tension of the viscous silicon nitride. Pores with radii in the range 1 to $5 \mathrm{~nm}$ can be directly drilled by the high intensity field emission electron beam, which breaks the covalent bond and causes $\mathrm{Si}$ and $\mathrm{N}$ to be sputtered away into the vacuum. The time taken to fabricate is about 1 minute for a $5 \mathrm{~nm}$ dia. nanopore and a $200 \mathrm{KeV}$ beam. Following surface tension model, the pore drilled by TEM can be further expanded or shrunk to the desired size at the specific range of intensities of electron beam (Fig. $2 \& 3$ ). These experiments were performed using a JEOL 2010 F (S)TEM. Parallelism achievable by fabrication of a high density nanopore array is possible by autonomous control of the (S)TEM system with interface to a lithography based pattern writing system. The TEM is an ideal platform for this approach by nature having superb intrinsic stability for high resolution imaging and precise beam control in STEM operation mode.

This new fabrication approach of solid-state nanopores utilizing a TEM opens up the possibility of the fine-tuning the diameter of the nanopores to unprecedented precision. By controlled manipulating of electron beam intensity, the shrinking and expanding process can be controlled until when the desired diameter has been reached. 


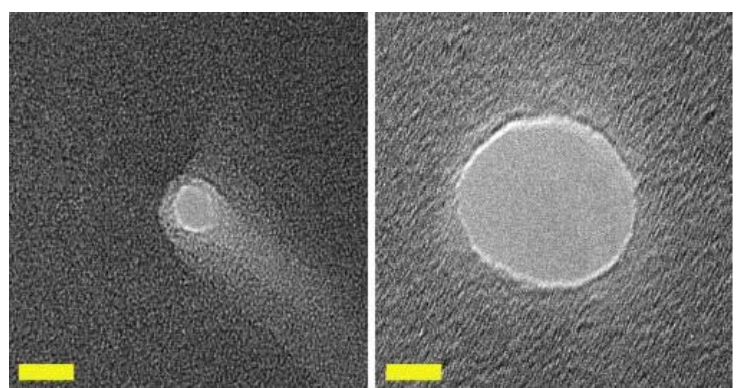

Fig. 1. $5 \mathrm{~nm}$ and $20 \mathrm{~nm}$ nanopores in Si3N4 membranes. The bar is $5 \mathrm{~nm}$.

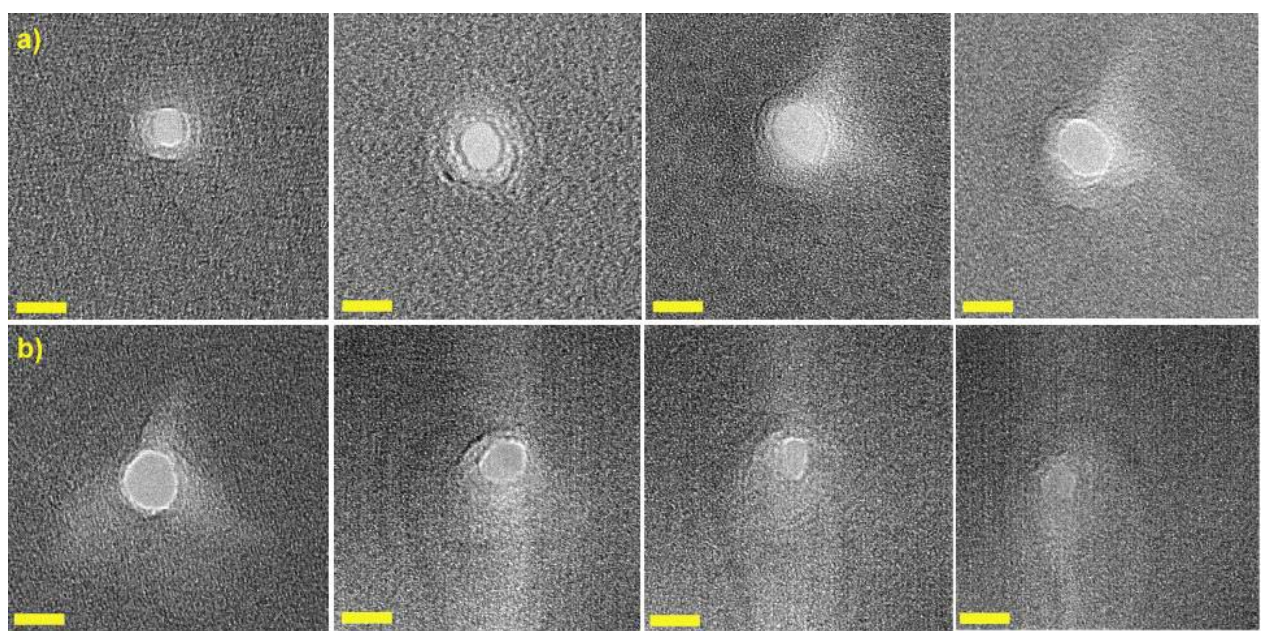

Fig. 2. Sequence of micrographs obtained during imaging of a nanopore in a TEM microscope. (a) drilling and (b) shrinking process. The bar is $5 \mathrm{~nm}$.
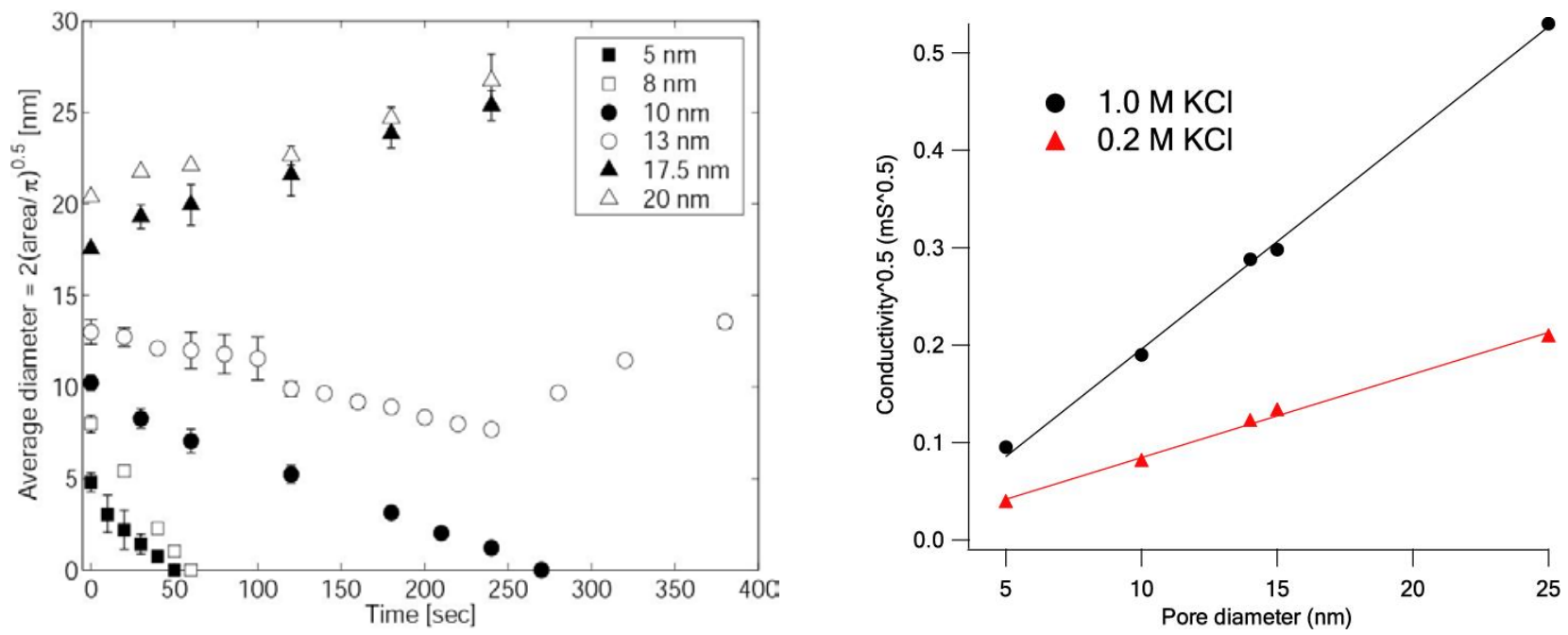

Fig. 3.(left) Diameter versus time for shrinking and expanding pores with various initial sizes of 5, $8,10,13,17.5$, and $20 \mathrm{~nm}$ when an intensity of electron beam fixed at $106 \mathrm{e} / \mathrm{nm}^{2}$.

Fig. 4. (right) Conductivity of the solid-state nanopores in $1.0 \mathrm{M}$ and $0.2 \mathrm{M} \mathrm{KCl}$ electrolyte $(+10$ $\mathrm{mM}$ TRIS buffer, adjusted to $\mathrm{pH} 8.5$ ) as a function of the pore diameter. 